\section{Mi Coming Events

2014

Neuroscience 2014

November 15-19, 2014

Washington, DC

www.sfn.org

EM Structure Determination

November 17-20, 2014

La Jolla, CA

http://nramm.scripps.edu/2014-workshop

Electron Tomography Conference November 17-20, 2014

Riviera Maya, Mexico

www.zingconferences.com/conferences/

electrontomography

\section{MRS Fall Meeting}

November 30-December 5, 2014

Boston, MA

www.mrs.org/fall2014

\section{ASCB Annual Meeting}

December 6-10, 2014

Philadelphia, PA

http://am.ascb.org/meetings

\section{5}

Quantitative Biolmaging Conference

January 7-9, 2015

Institute Pasteur, Paris, France

www.quantitativebioimaging.com

\section{MSC-SMC Annual Meeting}

May 26-29, 2015

Hamilton, Ontario, Canada

www.bimr.ca/events/msc-smc-annual-meeting-2015

Microscopy \& Microanalysis 2015

August 2-6, 2015

Portland, OR

www.microscopy.org

2016

Microscopy \& Microanalysis 2016

July 24-28, 2016

Columbus, $\mathrm{OH}$

www.microscopy.org

\section{7}

Microscopy \& Microanalysis 2017

July 23-27, 2017

St. Louis, MO

www.microscopy.org

\section{8}

Microscopy \& Microanalysis 2018

August 5-9, 2018

Baltimore, MD

www.microscopy.org

\section{9}

Microscopy \& Microanalysis 2019

August 4-8, 2019

Portland, OR

www.microscopy.org

More Meetings and Courses

Check the complete calendar near the back of this magazine.

\title{
Coronary Arteries Arise from Two Distinct Sources
}

\author{
Stephen W. Carmichael ${ }^{1 *}$ and Robert H. Anderson ${ }^{2}$ \\ ${ }^{1}$ Mayo Clinic, Rochester, MN 55905 \\ ${ }^{2}$ Institute of Genetic Medicine, Newcastle University, UK \\ *carmichael.stephen@mayo.edu
}

The development of coronary arteries that supply the heart muscle (myocardium) is a fundamental biologic question with profound ramifications for human health and disease. A better understanding of the embryology of these vessels could potentially lead to the development of therapies to repair damaged myocardium and reduce deaths from heart disease.

Although not rigorously tested, coronary vessels have been presumed to arise as a continued growth of embryonic vessels after an animal is born. Recently Xueying Tian, Tianyuan Hu, HuiZhang, Lingjuan He, Xiuzhen Huang, Qiaozhen Liu, Wei Yu, Liang He, Zhen Yang, Yan Yan, Xiao Yang, Tao Zhonu, William Pu, and Bin Zhou [1] have demonstrated in a postnatal mouse model that there is another source of coronary arteries that allows the rapidly enlarging heart to receive adequate vascular support (Figure 1).

Early in embryonic development, coronary vessels form as a vascular plexus that covers the outer surface of the heart. At this early stage the underlying ventricular walls are composed of a relatively thin compact layer and a much more extensive trabecular layer, the latter made up of multiple fingerlike projections into the ventricular cavities. With ongoing development, the compact myocardium develops coronary vessels from within, whereas the trabeculations directly exchanges nutrients with blood within the chamber without

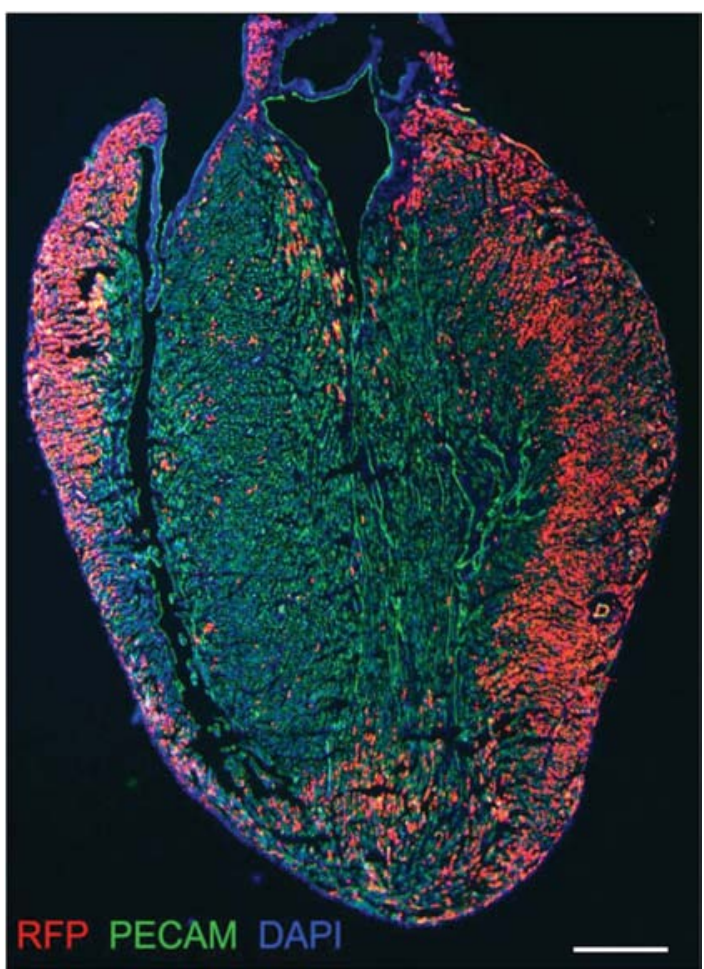

Figure 1: Section of tamoxifen-treated (at embryonic day 10.5) mouse heart at postnatal day 7 stained with red fluorescent protein (RFP), platelet endothelial cell adhesion molecule (PECAM), and 4',6-diamidino-2-phenylindole (DAPI) a well-known marker for nucleic acids. The RFP stains cells that arise from embryonic vessels, the cells stained with PECAM are bona fide coronary vessels that arise postnatally, and the DAPI stains nuclei. Scale bar is $0.5 \mathrm{~mm}$. requiring blood vessels. But prior to birth the trabecular myocardium coalesces with the outer compact myocardium and now requires its own blood supply. The mechanism for the ongoing postnatal vascularization of these newly developed compact ventricular walls has not been known.

Several different microscopic techniques were used to differetiate this second source of coronary arteries from the ones that originated prenatally. These include stereo microscopy, immunofluorescence microscopy, and confocal microscopy. Tian et al. first permanently labeled embryonic vascular endothelial cells (VECs). They found that at certain postnatal time periods these labeled cells were confined to the outer portions of the myocardial wall. Unlabeled endothelial cells accumulated in the inner portions of the wall. They were identified by positive markers for VECs, but clearly they were not derived from the embryonic vessels.

To define the developmental origin for the vessels formed $d e$ novo in the inner portions of the 


\section{Right results...Real-time}

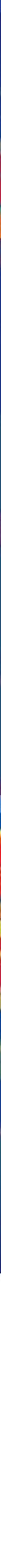

To see how to obtain the right result visit www.oxford-instruments.com/results

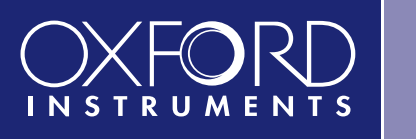

The Business of Science ${ }^{\circledast}$ 
heart wall, Tian et al. developed a strain of mice that allowed embryonic endothelial cells to be labeled distinctly from the cells that developed postnatally. A series of ingenious experiments showed that in the inner myocardial wall, VECs arise by lineage conversion of neonatal endocardial cells. The interventricular septum, like the inner myocardial wall, is also vascularized by lineage conversion of endocardial cells to coronary VECs. The authors suggest that there had been postnatal "compaction" of the trabeculations to form the innermost layer of myocardium. This aspect remains controversial. Tian et al. nonetheless offer compelling evidence that this inner layer is supplied by vessels that arise de novo, and not from the embryonic VECs

This newly discovered dual origin of coronary arteries presents a new mechanism for a rapidly developing functional vascular supply of the myocardium. This novel discovery will need to be verified and the responsible molecular mechanisms elucidated. It will also need to be established whether the trabecular layer of the myocardium does, indeed, compact to form the inner part of the ventricular walls. Still, the findings regarding the formation of the coronary vasculature are not only an exciting addition to our knowledge of heart development, but have important implications for cardiac diseases and cardiac regenerative medicine!

\section{References}

[1] X Tian et al., Science 345 (2014) 90-94.

[2] The authors gratefully acknowledge Dr. Bin Zhou for reviewing this article.

\section{PELCO'silicon Nitride} \& Silicon Dioxide Membranes

Next Generation SiN TEM Support Films

- Robust and clean 8, 15, 50 and $200 \mathrm{~nm}$ SiN substrates

- $63.0 \mathrm{~mm}$ frame

- EasyGrip ${ }^{\text {Tm }}$ edges

- Free from debris

- Super flat 8, 15, and 40nm silicon dioxide substrates
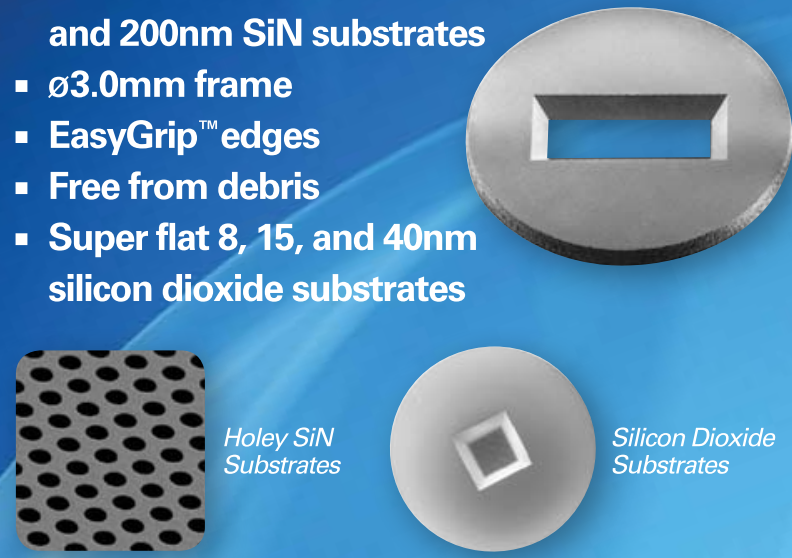

$$
\begin{aligned}
& \square \text { MED PELLA, MV } \\
& \text { Microscopy Products for Science and Industry }
\end{aligned}
$$

www.tedpella.com sales@tedpella.com 800.237.3526
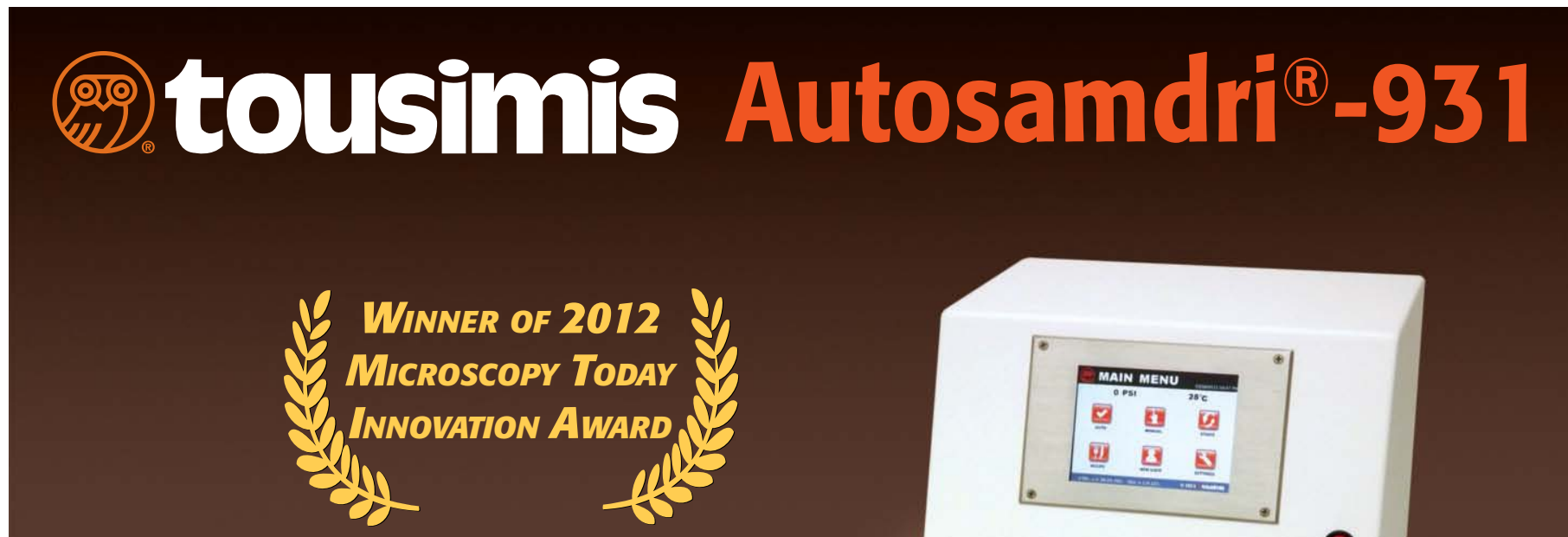

MUth-Application Critical Point Dryer

- Patent Pending "Stasis Softurare"

- Available in 1.25", $2.50^{\circ}$ and 3.40 I.D. Chamber stizes

- Made in U.S.A.

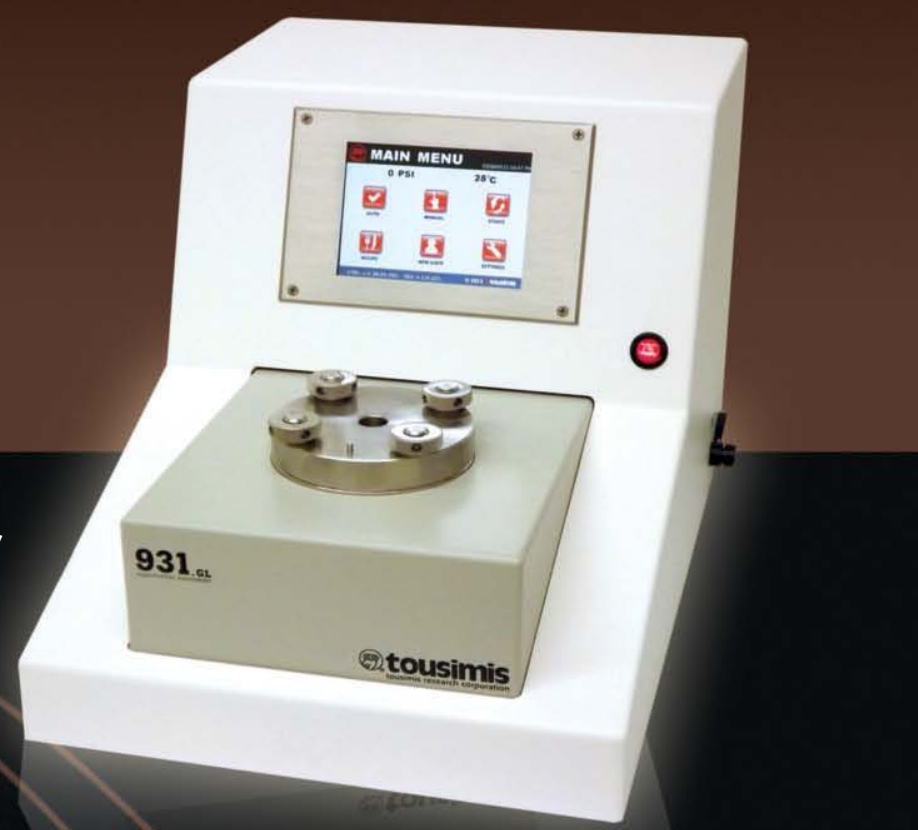


